\title{
DEPRESSION IN PATIENTS WITH HEART FAILURE
}

\author{
Mariya Georgieva ${ }^{1}$, Lilyana Mircheva $^{2}$, Yoto Yotov ${ }^{2}$ \\ ${ }^{1}$ Department of Nursing Care, Faculty of Public Health, Medical University of Varna \\ ${ }^{2}$ First Department of Internal Diseases, Faculty of Medicine, Medical University of Varna
}

\begin{abstract}
INTRODUCTION: Heart failure (HF) is a chronic progressive syndrome, which is a result of preceding heart diseases and manifests with serious symptoms. Depression and anxiety are frequent comorbidities in chronic HF, which deteriorate the general status of the patients and are related to unfavorable health results-increased number of hospitalizations, deteriorated quality of life, and elevated mortality.

AIM: The aim of this article is to assess the tendency to fall into depression of patients with HF in NYHA class III-IV, hospitalized in the non-invasive cardiology clinic of St. Marina University Hospital, Varna and the link to socioeconomic factors-social isolation, family status, educational level, region of residence and so on.

MATERIALS AND METHODS: Overall, 39 patients with HF (17 women and 22 men) were investigated. They were in III-IV NYHA functional class during hospitalization. After preliminarily informed consent, they were asked to fill out a validated screening questionnaire for depression in adults-the 15 -item Geriatric Depression Scale (GDS). The link to the patients' socioeconomic, demographic, biological, and clinical characteristics was sought with the help of a multi-factor linear and logistic regression analysis.

RESULTS: The results for the level of depression among HF patients were related to socioeconomic factorsregion of residence, level of education, social isolation as well as to other variables-gender, age, and systolic left ventricular function assessed through the ejection fraction (EF). Higher levels of depression were observed in patients who resided in larger populated areas, with higher level of education and patients living alone. As far as gender was concerned, females were more susceptible to depressive episodes than males. At an average EF of $49.54 \pm \mathbf{1 5 . 2 9 \%}$, the decreased left ventricular systolic function was also a factor of the degree of development of anxiety and depression. In a multi-factor analysis, however, only living alone was related to enhanced depressive attitude.
\end{abstract}

CONCLUSION: Depression/anxiety in HF patients requires increased need of health care, shows poor health results, has high costs for treatment related to the serious symptoms, disability and high mortality. Regardless of that, no programs for timely detection, diagnosis, and treatment have been developed yet.

Keywords: depression, heart failure, lifestyle, socioeconomic factors

\footnotetext{
Address for correspondence:

Mariya Georgieva

Faculty of Public Health

Medical University of Varna

55 Marin Drinov St

9002 Varna

e-mail:mariq_velina@abv.bg
}

Received: November 18, 2020

Accepted: December 6, 2020

\section{INTRODUCTION}

Heart failure (HF) is a progressively developing clinical syndrome, observed predominantly in people at the age of 65 years or older (1-3). In this age group, this is the leading cause for cardiovascular disease morbidity and mortality. About $50 \%$ of the patients die within up to 5 years after determining the diagnosis (4). HF has a more unfavorable prognosis than a number of cancers in both genders (5). The 
progression of HF is accompanied by aggravation of the symptoms, especially when combined with concomitant pathology. Depression is one of the many comorbidities, which exert significant impact on the symptoms and the development of HF. It contributes to frequent re-hospitalizations and deteriorates the quality of life (6).

Depression and anxiety are the most frequently encountered pathological and psychological states in HF patients (7-9). Chronic diseases alone are the main reason for the development of a depressive syndrome. The limitations imposed by the disease, the exacerbation of the symptoms of the main disease, the frequent hospitalizations are major factors in the development of the symptoms of depression.

Depression and anxiety, added to the symptoms of HF, deteriorate the quality of life in the patients, limit their physical activity, make the carrying out of daily activities difficult and are the reason for social isolation and disability (10). This generates many health, social and economic problems amongst bedridden patients in particular. Patients with HF and depression are at an increased risk of death or secondary cardiac events $(8,11-14)$.

There is a considerable overlapping in the symptoms of HF and depression: sense of shortness of breath, fatigue and exhaustion, irritability, hopelessness, lack of desire for social life, lack of appetite, change in the body weight, sleep disturbances, absence of interest in or pleasure from activities and hobbies, lack of desire and fear of physical activity, troubled concentration and memory (15).

That is why it is important to determine the link between depression/anxiety and HF and the prevalence of depression amongst aging patients with HF for timely diagnosis, follow-up and treatment of this high-risk population.

\section{AIM}

The aim of this article is to screen for possible depression in elderly patients hospitalized for NYHA class III-IV HF, and to investigate the link to the socioeconomic demographic and clinical factors.

\section{MATERIALS AND METHODS}

We assessed 39 patients (17 females and 22 males) who were hospitalized in the Second Cardiology Clinic of St. Marina University Hospital, Var- na, Bulgaria. All patients were in NYHA class III and IV at admission. After giving informed consent, all of them who were mentally adequate enough to execute questionnaires were asked to fill in the 15item Geriatric Depression Scale (GDS) (16). This tool is validated as a screening test for depression in elderly populations. According to the points on the scale, we divided the patients into three groups: bellow 5 points - no signs of depression; from 6 to $10-$ possible depression, and if the score was $>10$ points the depression was almost definite. Further, we dichotomized the GDS score in present ( $>5$ points) or absent ( $<5$ points) depression. We related the depression scores with various socioeconomic factors-social deprivation and living alone, level of education, region of living, as well as with other variables, such as age, gender, and systolic left ventricular (LV) function. The latter was evaluated through ejection fraction (EF), which was measured by the standard Simpson's multidisc method at a 4-chamber view on transthoracic echocardiography.

For continuous variables, we tested the null hypothesis using the independent t-test or one-way analysis of variance (ANOVA), where appropriate. The results were given as mean \pm standard deviation or median in case of abnormal distribution. For categorical variables, the data were presented as numbers and percentage, and the chi-square test or Fisher's exact test (if small numbers) was applied. In order to test the independent effect of the various covariates on the GDS score, we used regression analyses. First, we applied linear multivariate regression analysis with the GDS points as a continuous outcome variable and age, gender, living alone, place of living, and education as predictor variables. All variables were entered into the model, with $p<0.05$ to stay in the model and $\mathrm{p}<0.1$ to be removed from it. Then, we tested the association between presence of depression as categorical variable (yes/no) and the $\mathrm{EF}$ as dichotomous variable $(>40 \% /<40 \%)$, social isolation, region of living, education, sex, and age as independent variables in logistic regression analysis. The backward stepwise selection was used with Wald's method for testing of significance. The significance was accepted if $\mathrm{p}<0.05$. 


\section{RESULTS}

In total, 39 patients with NYHA class II-IV HF were investigated. The average age of the group was $72.95 \pm 6.03$ years, range from 64 to 92 years. The average $\mathrm{EF}$ of the left ventricle was $49.54 \pm 15.29 \%$ (19-79\%). Eighteen (46.2\%) individuals were with $\mathrm{EF}<50 \%$ and $11(21.8 \%)$ were with EF below $40 \%$. The gender distribution of the participants was: 22 (56.4\%) males and 17 (43.6\%) females.

The main demographic characteristics were as follows: 17 (43.6\%) were with primary school education, and 22 (56.4\%) were with secondary school education. Twenty-three (59\%) resided in a city/town, and 16 (41\%) were from rural regions. Family status: The majority lived with a partner-22 (56.4\%), and 17 (43.6\%) were widowed or divorced. Baseline data with presence of probable and definite depression are shown in Table 1.
The mean number of points from the questionnaire for depressive attitude was $7.23 \pm 3.12$ (range $1-13$ ), and the median was 7.00 points. The probability for development of depressive symptoms is presented on Fig. 1. Only $17.9 \%$ of the hospitalized for HF were with $<5$ points (without depression).

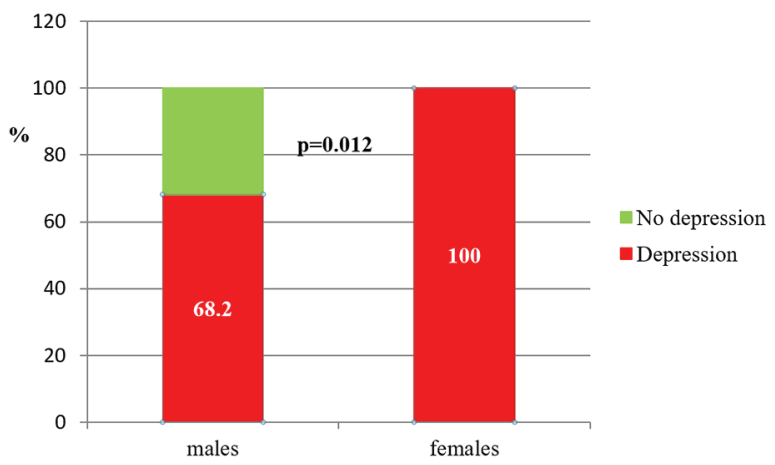

Fig. 2. Depression rates by gender categories

Table 1. Main characteristics of the participating elderly patients with HF $(n=39)$ according to their GDS result for presence of depression

\begin{tabular}{|c|c|c|c|c|c|}
\hline & Total $(n=39)$ & $\begin{array}{l}\text { No depression } \\
\leq 5 \mathrm{p}(\mathrm{n}=7)\end{array}$ & $\begin{array}{c}\text { Possible } \\
\text { depression } \\
\text { 6-9 p }(n=21)\end{array}$ & $\begin{array}{l}\text { Definite depression } \\
\quad \geq 10 \mathrm{p}(\mathrm{n}=11)\end{array}$ & $\mathbf{P}$ \\
\hline Age years (mean $\pm S D)$ & $72.95 \pm 6.03$ & $74.86 \pm 4.14$ & $71.67 \pm 7.18$ & $74.18 \pm 4.14$ & 0.36 \\
\hline $\mathrm{EF} \%($ mean $\pm \mathrm{SD})$ & $49.54 \pm 15.29$ & $41.00 \pm 15.43$ & $52.48 \pm 16.26$ & $49.36 \pm 12.17$ & 0.23 \\
\hline Male gender (\%) & $22(56.4)$ & $7(100)$ & $7(33.3)$ & $8(72.7)$ & 0.004 \\
\hline Secondary school (\%) & $17(43.6 \%)$ & $2(28.6)$ & $9(42.9)$ & $6(54.5)$ & 0.55 \\
\hline Living in city/town (\%) & $23(59)$ & $2(28.6)$ & $14(66.7)$ & $7(63.6)$ & 0.19 \\
\hline Living alone (\%) & $17(43.6)$ & $1(14.3)$ & $9(42.9)$ & $7(63.6)$ & 0.12 \\
\hline
\end{tabular}

\section{Depression moods in \%}

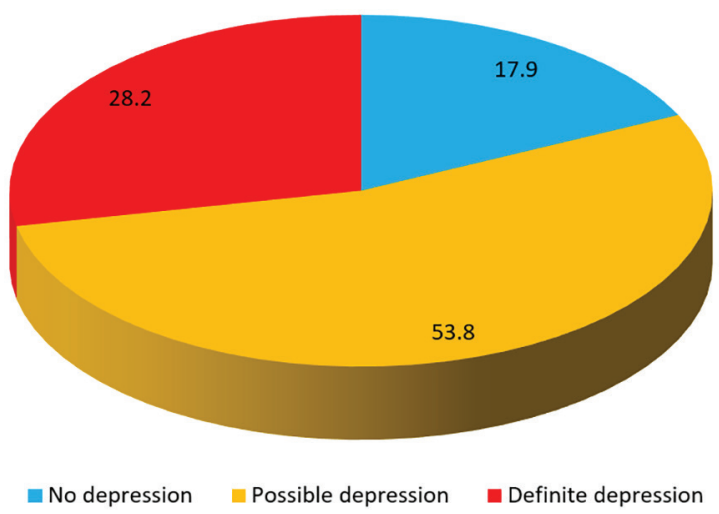

Fig. 1. Distribution of patients with heart failure according to the probability of development of depression
Females were more prone to senile depression than males (Fig. 2), p=0.012 according to Fisher's exact test.

There was no significant association between the dichotimized variable presence of depression yes/no and living alone, place of living, and level of education. The resuls were also insignificant for age and LV systolic function (Table 1).

\section{REGRESSION ANALYSIS}

\section{Linear Regression Analysis}

The model included GDS points as a dependent continuous variable and several independent 
Depression in patients with heart failure

Table 2. Linear multivariate regression analysis of GDS points as an outcome variable and several important independent factors

\begin{tabular}{|c|c|c|c|c|c|c|}
\hline \multirow[t]{2}{*}{ Model } & \multicolumn{2}{|c|}{ Unstandardized Coefficients } & \multirow[t]{2}{*}{$\mathrm{T}$} & \multirow[t]{2}{*}{ Sig. } & \multicolumn{2}{|c|}{$\begin{array}{l}\text { 95.0\% Confidence Interval } \\
\text { for B }\end{array}$} \\
\hline & Beta & Std. Error & & & Lower Bound & Upper Bound \\
\hline (Constant) & 12.361 & 6.639 & 1.862 & 0.072 & -1.163 & 25.884 \\
\hline Gender & -1.120 & 1.177 & -0.952 & 0.348 & -3.518 & 1.277 \\
\hline Age & -0.040 & 0.088 & -0.458 & 0.650 & -0.220 & 0.139 \\
\hline Living alone & 2.298 & 1.097 & 2.096 & 0.044 & 0.065 & 4.532 \\
\hline Place of living & -1.413 & 1.308 & -1.080 & 0.288 & -4.078 & 1.253 \\
\hline Education & -0.242 & 1.207 & -0.200 & 0.843 & -2.701 & 2.218 \\
\hline $\mathrm{EF} \%$ & 0.011 & 0.038 & 0.292 & 0.772 & -0.066 & 0.088 \\
\hline
\end{tabular}

a. Dependent variable: Points of depression

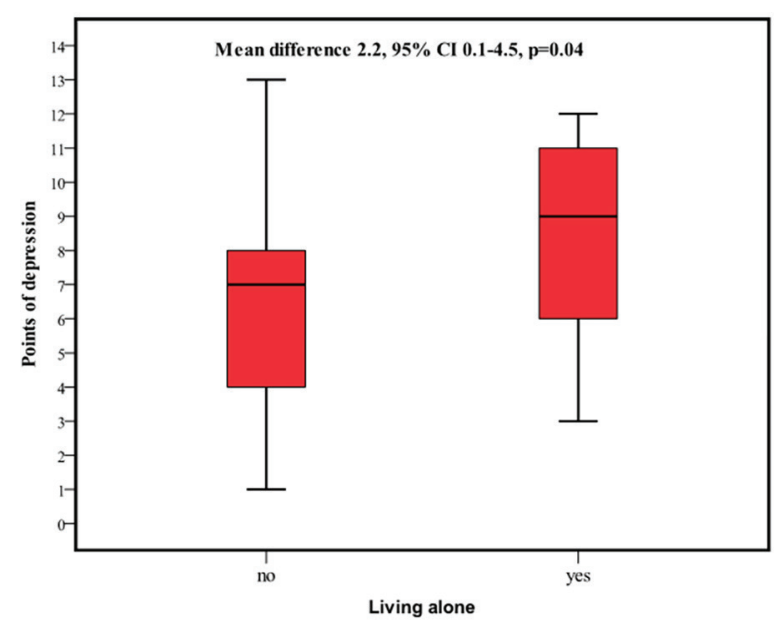

Fig. 3. Box-and-whiskers plot of the mean difference in the GDS score points between elderly people with HF living alone or with someone predictors, all of them simultaneously included in the model. The results are presented in Table 2.

The only significant factor with an effect on depression was living alone. The mean difference was 2.3 points more if the elderly person lived alone, 95\%CI 0.1-4.53, $\mathrm{p}=0.044$ (Fig. 3).

\section{Logistic Regression Analysis}

No single indicator was a significant predictor of GDS score $>5$ points. All variables in the model were insignificant (Table 3).

\section{DISCUSSION}

The occurrence of depression in HF patients is related to worse clinical results. At an attempt for therapeutic influence and improvement of the general status, the symptoms of anxiety and depression (despair, denial, lack of motivation, doubt in the fa-

Table 3. Logistic regression analysis with outcome variable the GDS score $>5$ points and predictor variables age, gender, social isolation (living alone vs. living with someone), educational level (primary, secondary school, and university), place of living (urban vs. rural), EF as categorical (reduced vs. preserved)

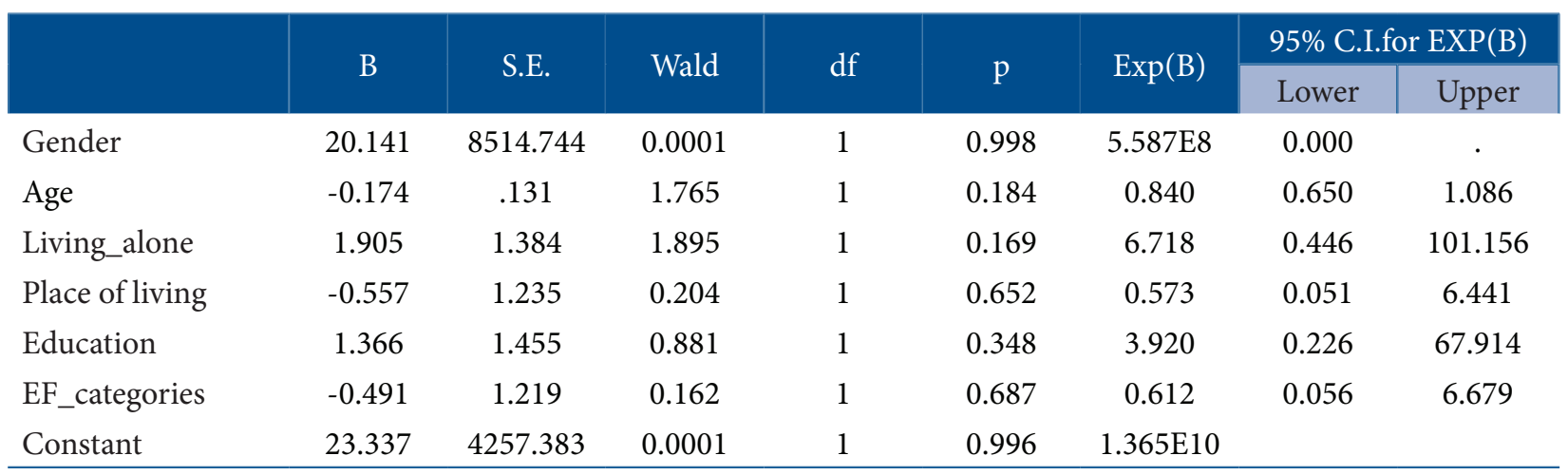


vorable course of the disease) result in nonadherence to the treatment and in poor persistence to the therapeutic guidelines, which additionally deteriorates the general status of the patients. This is why it is of special significance to determine the frequency of depression and anxiety among them.

In our study, we found significant prevalence of depressive attitudes among elderly HF patients $-28 \%$ were with depression as a most probable diagnosis and another $54 \%$ - as a very possible one. A number of clinical studies show the presence of clinically significant depression in HF patients. The prevalence varies between 9 and $60 \%$ depending on the diagnostic tools (17). One meta-analysis of 37 studies in patients with HF indicates that, as a percentage, depression is more frequent among patients with HF than in the general population. The clinically significant symptoms affect $21.5 \%$ of the HF patients reporting a depressive syndrome on the basis of questionnaires and $19 \%$ - on the basis of a diagnostic interview (8). The reasons for this variability of depression prevalence in HF are several: 1) the methods used for depression assessment (questionnaire vs. structured interview); 2) the armamentarium for depression diagnosis-many instruments with varying complexity; 3) conservative vs. more liberal discriminative cut-off values for defining depression; 4) the HF severity, the mean age, ethnic origin and sex of the patients searched; 5) hospitalized or ambulatory patients.

Females were significantly more prone to depressive moods than males in our sample-100\% vs. $68 \%, p=0.012$. Our data on this sex difference are confirmed by other studies. In the meta-analysis mentioned before, T. Rutledge et al. (8) found that clinical depression was present in $32.7 \%$ of the female patients (range 11-67\%) vs. $26.1 \%$ (range 7-63\%) in the male subgroup. Regarding gender, it has been shown that women also experience more intense depression (18). In another study (19) of 155 HF patients NYHA class II-IV with reduced ejection fraction $(\mathrm{EF}<40 \%)$ the women were more likely (64\%) to be depressed than men (44\%). On the contrary, SA Thomas et al. (20) showed that men with HF were more likely to become depressed than the general population. The independent effect of sex on depressive predisposition, however, was lost in our multivariable regression analyses, both linear and logistic. The possible explanations are the small number of patients or that the gender difference is mediated through other social factors, like education, living conditions, and social isolation.

Our results showed that social isolation and living alone were important factors in the relation depression/heart failure. The relative share of the patients with HF in our group who live alone and without social support increased steadily from $14 \%$ in those without depression to more than $63 \%$ in those with most probable depression. Moreover, the link between social isolation and depression remained the only significant factor in the linear multivariate regression analysis, with a mean difference in GDS score of more than 2 points. The same results were reported in a big community study in patients with HF where depression prevalence increased from $14 \%$ in the group with low perceived social isolation to $52 \%$ in those with high isolation (21). The social isolation increased mortality risk $>3$ times, hospitalizations were higher by $70 \%$, and the risk of emergency department visits went up by $57 \%$ in this high-risk population. Other studies, including one meta-analysis, have reinforced the role of loneliness and social isolation as a risk factor for cardiovascular diseases $(22,23)$. This was not confirmed by others (11) where the marital status was not different between various levels of depression in 402 patients with HF.

Other socioeconomic factors, like education and place of living, did not show any significant link to the tendency to suffer from depression in our patients with HF. Similar lack of association between social economic status, lower level of education, and depression was reported by I Zahid et al. (24). They assessed $170 \mathrm{HF}$ patients with $\mathrm{EF}<40 \%$, at a mean age 64 years and $73 \%$ males. Almost two thirds (60\%) had some level of depression assessed by a PHQ-9 questionnaire. Low economic status had $61.8 \%$ of the depressed patients and $60.3 \%$ of those without depression, $\mathrm{p}=0.84$. No education characterized 30.4 and $32.4 \%$, respectively, $\mathrm{p}=0.78$. M Polikandrioti et al. (19) report a trend to observe higher depression scores in low-educated patients with HF. In their group of 139 patients with HF primary school education was insignificantly related to depression compared to high education level $(\mathrm{p}=0.05)$. Unlike us, however, they found that living in a metropolitan area predisposed to higher risk of depression com- 
pared to living in villages $(\mathrm{p}=0.021)$. One possible explanation of these findings is that in our study we did not measure socioeconomic status directly. Instead, we used proxies like education level and urban/rural environment. Although they reflect some features of the socioeconomic condition, the importance of the position in the society is more complex than just those two factors. However, education and socioeconomic factors are important for the well-being, since HF patients do not easily accept the disease and adopt non-compliance to drug therapy (25).

The severity of HF is assessed either by the reduced systolic function measured by EF or by the impaired functional capacity measured by the NYHA functional class. Several meta-analyses and individual studies show that severity of depression correlates with higher NYHA class $(8,26)$. All our patients were in NYHA class III and IV when they were hospitalized. As they were with advance stages of HF, it was not possible to look for the effect of severity of both diseases. Our population of HF patients was a mixture of both reduced and preserved EF, with mean EF 49\%. Only one fifth of the patients were with $\mathrm{EF}<40 \%$. But it is well known that the quality of life and psychological well-being are not always tightly following the severity of the disease. The lack of relation between EF and GDS score was also observed by $\mathrm{V}$ Vaccarino et al. (27) In their cohort of 391 patients with $\mathrm{HF}$, almost $50 \%$ were with $\mathrm{EF}>40 \%$ and the mean GDS score was not different in the various levels of EF reduction ( $\mathrm{p}=0.13)$. The mean GDS score was almost identical to ours-7.3 vs. 7.23. Interestingly, they also found a trend of higher depression tendency in the females $(p=0.09)$ but in their sample the low-education group showed significantly higher scores than their more educated peers-mean 7.81 vs. 6.92, $\mathrm{p}=0.004$. Marital status had no effect on depression in this study. On the contrary, van Melle et al. (28) found significant inverse relation between $\mathrm{EF}$ and the development of depression symptoms $(\mathrm{p}<0.01) . \mathrm{EF}<30 \%$ predicted 4.46 times higher risk of clinically evident depression than $\mathrm{EF}>60 \%$ in postmyocardial infarction patients 3 months after the incident event. However, these were patients with acute myocardial infarction (MI) and not with HF. Interestingly, the presence of depression steeply diminished with time and was $11 \%$ at 6 months and $5 \%$ at one year after MI. Thus, the extrapolation of the re- sults to patients with congestive heart failure (CHF) may be misleading, as the chronic condition has a different course pattern than the acute one.

This study has its limitations as well. First, the comorbidity of the monitored patients was not clarified, the availability of other chronic diseases except $\mathrm{HF}$, as this is an additional burden for occurrence of clinical and psychological symptoms. Moreover, the significant prevalence of depression among HF patients may to a certain degree be explained with the availability of accompanying comorbidities. Second, the comparison with other similar studies is rather difficult, sometimes even impossible, as various criteria of diagnosis and evaluation of depression are used. Third, a relatively small number of patients, hospitalized in a single center in referral university hospital, is evaluated. There are reports on some differences in the levels of depression and anxiety between hospitalized patients and outpatients. This is, to a certain degree, a predictor of psychological and behavioral attitudes amongst patients. That is why, our study may be the basis to conduct similar research in other hospital centers, as well as in ambulatory patients. The role of the healthcare specialists in the latter research may be extremely important and with substantial significance.

\section{CONCLUSIONS}

The early recognition of depression in cardiac pathology is of important significance for the clinical practice, for an improvement in the health status and the quality of life. If depression is recognized and adequately treated, the general condition and the prognosis of HF patients will improve. Hospitalizations will decrease, the quality of life of the patients will improve, the health and economic results will be optimized.

That is why it is important to provide complex health care, which must include screening for risk factors, a control over the symptoms of deterioration, guidelines-oriented treatment and psychological cooperation and support.

\section{REFERENCES}

1. Go AS, Mozaffarian D, Roger VL, Benjamin EJ, Berry JD, Blaha MJ, et al. Heart disease and stroke statistics--2014 update: a report from the American 
Heart Association. Circulation. 2014;129(3):e28e292. doi: 10.1161/01.cir.0000441139.02102.80.

2. Mosterd A, Hoes AW. Clinical epidemiology of heart failure. Heart. 2007; 93(9):1137-46. doi: 10.1136/hrt.2003.025270.

3. Redfield MM, Jacobsen SJ, Burnett JC, Mahoney DW, Bailey KR, Rodeheffer RJ. Burden of systolic and diastolic ventricular dysfunction in the community: appreciating the scope of the heart failure epidemic. JAMA. 2003; 289(2):194-202. doi: 10.1001/jama.289.2.194.

4. McMurray JJ, Stewart S. The burden of heart failure. Eur Heart J. 2002; 4(suppl D):D50-8.

5. Mamas MA, Sperrin M, Watson MC, Coutts A, Wilde K, Burton C, et al. Do patients have worse outcomes in heart failure than in cancer? A primary care-based cohort study with 10-year follow-up in Scotland. Eur J Heart Fail. 2017; 19(9):1095-104. doi: 10.1002/ejhf.822.

6. Coelho R, Ramos S, Prata J, Bettencourt P, Ferreira A, Cerqueira-Gomes M. Heart failure and health related quality of life. Clin Pract Epidemiol Ment Health. 2005;1:19. doi: 10.1186/1745-0179-1-19.

7. Celano CM, Villegas AC, Albanese AM, Gaggin HK, Huffman JC. Depression and anxiety in heart failure: a review. Harv Rev Psychiatry. 2018;26(4):175-84. doi: 10.1097/ HRP.0000000000000162.

8. Rutledge T, Reis VA, Linke SE, Greenberg BH, Mills PJ. Depression in heart failure: a meta-analytic review of prevalence, intervention effects, and associations with clinical outcomes. J Am Coll Cardiol. 2006; 48(8):1527-37. doi: 10.1016/j. jacc.2006.06.055.

9. Daskalopoulou M, George J, Walters K, Osborn DP, Batty GD, Stogiannis D, et al. Depression as a risk factor for the initial presentation of twelve cardiac, cerebrovascular, and peripheral arterial diseases: Data Linkage Study of 1.9 million women and men. PLoS One. 2016;11(4):e0153838. doi: 10.1371/journal.pone.0153838.

10. Sullivan M, Levy WC, Russo JE, Spertus JA. Depression and health status in patients with advanced heart failure: a prospective study in tertiary care. J Card Fail. 2004; 10(5):390-6. doi: 10.1016/j. cardfail.2004.01.011.

11. Moraska AR, Chamberlain AM, Shah ND, Vickers KS, Rummans TA, Dunlay SM, et al. Depression, healthcare utilization and death in heart failure: a community study. Circ Heart Fail. 2013;6(3):387-94. doi: 10.1161/ CIRCHEARTFAILURE.112.000118.

12. Adelborg K, Schimidt M, Sundbøll J, Pedersen L, Videbech P, Bøtker HE, et al. Mortality risk among heart failure patients with depression: A nationwide population-based cohort study. J Am Heart Assoc. 2016;5(9):e004137. doi: 10.1161/ JAHA.116.004137.

13. Fan $\mathrm{H}, \mathrm{Yu} \mathrm{W}$, Zhang Q, Cao H, Li J, Wang J, et al. Depression after heart failure and risk of cardiovascular and all-cause mortality: a meta-analysis. Prev Med. 2014;63:36-42. doi: 10.1016/j. ypmed.2014.03.007.

14. Frasure-Smith N, Lespérance F, Habra M,Talajic M, Khairy P, Dorian P, et al. Elevated depression symptoms predict long-term cardiovascular mortality in patients with atrial fibrillation and heart failure. Circulation. 2009;120(2):134-40. doi: 10.1161/CIRCULATIONAHA.109.851675.

15. Pintor L. Heart failure and depression, an often neglected combination. Rev Esp Cardiol. 2006; 59(8):761-5.

16. Yesavage JA, Brink TL, Rose TL, Lum O, Huang $\mathrm{V}$, Adey MB, et al. Development and validation of a geriatric depression screening scale: A preliminary report. J Psychiatr Res. 1983; 17(1):37-49. doi: 10.1016/0022-3956(82)90033-4.

17. Mbakwem A, Aina F, Amadi C. Depression in patients with heart failure: is enough being done? Card Fail Rev. 2016;2(2):110-2. doi:10.15420/ cfr.2016:21:1.

18. Gottlieb SS, Khatta M, Friedmann E, Einbinder L, Katzen S, Baker B, et al. The influence of age, gender and race on the prevalence of depression in heart failure patients. J Am Coll Cardiol. 2004; 43(9):1542-9. doi: 10.1016/j.jacc.2003.10.064.

19. Polikandrioti M, Christou A, Morou Z, Kotronoulas G, Evagelou H, Kyritsi H. Evaluation of depression in patients with congestive failure. Health Sci J. 2010; 4(1):37-47.

20. Thomas SA, Friedmann E, Khatta M, Cook LK, Lann AL. Depression in patients with heart failure: physiologic effects, incidence, and relation to mortality. AACN Clin Issues. 2003;14(1):3-12. doi: 10.1097/00044067-200302000-00002.

21. Manemann SM, Chamberlain AM, Roger VL, Griffin JM, Boyd CM, Cudjoe TKM, et al. Perceived social isolation and outcomes in patients with heart 
failure. J Am Heart Assoc. 2018; 7(11):e008069. doi: 10.1161/JAHA.117.008069.

22. Valtorta NK, Kanaan M, Gilbody S, Ronzi S, Hanratty B. Loneliness and social isolation as risk factors for coronary heart disease and stroke: systematic review and meta-analysis of longitudinal observational studies. Heart. 2016; 102(13):1009-16. doi: 10.1136/heartjnl-2015-308790.

23. Xia N, Li H. Loneliness, social isolation, and cardiovascular health. Antioxid Redox Signal. 2018; 28(9):837-51. doi: 10.1089/ars.2017.7312.

24. Zahid I, Baig MA, Gilani JA, Waseem N, Ather S, Farooq AS, et al. Frequency and predictors of depression in congestive heart failure. Indian Heart J. 2018; 70(Suppl.3):S199-S203. doi: 10.1016/j. ihj.2018.10.410.

25. Lefteriotis C. Depression in heart failure patients. Health Sci J. 2013; 7(4):349-55.
26. Faller H, Steinbuchel T, Stoerk S, Scholwater M, Ertl G, Angermann C. Impact of depression on quality of life assessment in heart failure. Int J Cardiol. 2010; 142(2):133-7. doi: 10.1016/j. ijcard.2008.12.093.

27. Vaccarino V, Kasl SV, Abramson J, Krumholz HM. Depressive symptoms and risk of functional decline and death in patients with heart failure. J Am Coll Cardiol. 2001;38(1):199-205. doi: 10.1016/ s0735-1097(01)01334-1.

28. van Melle JP, de Jonge P, Ormel J, Crijns HJ, van Veldhuisen DJ, Honig A, et al. MIND-IT Investigators. Relationship between left ventricular dysfunction and depression following myocardial infarction: data from the MIND-IT. Eur Heart J. 2005;26(24):2650-6. doi: 10.1093/eurheartj/ehi480. 